\title{
Case report: MPN-eo with balanced rearrangement of MPRIP-PDGFRB t(5;17)(q32;p11), responding to low dose imatinib
}

\author{
Karolien Beel, Geneviève Ameye, Wim De Kelver, Lucienne Michaux \\ Centre of Human Genetics, University Hospitals Leuven and KU Leuven, Leuven, Belgium
}

Received: June 10, 2021

DOI: $10.5430 /$ dcc.v8n3p1
Accepted: July 8, 2021

URL: https://doi.org/10.5430/dcc.v8n3p1
Online Published: August 24, 2021

\begin{abstract}
We present a young patient with a rare eosinophilic myeloproliferative neoplasm MPN-eo with a balanced rearrangement of MPRIP-PDGFRB t(5;17)(q32;p11). This fusion gene was reported in only one other case in 2015. A personalized molecular test was developed with the diagnostic material of the patient. Our patient is in complete cytogenetic and molecular remission under low dose imatinib, two years after treatment initiation.
\end{abstract}

Key Words: Eosinophilia, MPN, PDGFRB and MPRIP, Imatinib

\section{CASE REPORT}

A 37-year-old man of North-African descent was referred to our Haematology Department with leukocytosis of $12.9 \times$ $10^{6} / \mathrm{L}$, marked eosinophilia of $5,110 / \mu \mathrm{l}$ and mild anemia with $\mathrm{Hb} 11.2 \mathrm{~g} / \mathrm{dl}$, detected by routine control. White blood cell count differentiation showed increased myelocytes by $3.6 \%$, metamyelocytes by $1.8 \%$, and vitamin B12 > 2,000 ng/L. The patient reported an allergy to pollen with mild symptoms of hay fever, which were treated with antihistamines. His IgE level was elevated to $1.75 \mathrm{kU} / \mathrm{L}$ (normal value < $0.35)$. In retrospect, blood results two years before already showed eosinophilia of 2,568/ $\mu \mathrm{l}(0-500)$ and a vitamin B12 level > 2,000 ng/L, with normal hemoglobin and leukocyte counts. His bone marrow aspirate morphology was normal and molecular testing of $B C R-A B L$ and FIPIL1-PDGFRA were negative.

Cytogenetic results showed a pseudodiploid karyotype with abnormal 5q. Subsequent FISH analysis showed a balanced rearrangement of the locus $P D G F R B / 5 q 32$ in $73 \%$ of the nuclei and in 5/6 metaphases. In the abnormal metaphases, the 5' telomeric signal was translocated to $\operatorname{der}(17) \mathrm{t}(5 ; 17)$, and the 3' centromeric signal remained on $\operatorname{der}(5) \mathrm{t}(5 ; 17)$ (see Figure 1). Cytogenetic results described according to the International System for Human Cytogenetic Nomenclature (ISCN 2020): 46,XY,del(5)(q32q34), add(17)(p11)[9]/46, $\mathrm{XY}$ [1].ish $\mathrm{t}(5 ; 17)(\mathrm{q} 32 ; \mathrm{p} 11)(3$ 'PDGFRB+,5'PDGFRB;5'PDGFR+)[5].nuc ish(PDGFRBx2) (3'PDGFRB sep 5'PDGFRBx1)[145/200]. The diagnosis of a myeloid neoplasm with eosinophilia and gene PDGFRB rearrangement (WHO 9966/3) was made. Sequencing analysis revealed the fusion partner of $P D G F R B$ and showed the presence of an $M P R I P$ (17p exon20)-PDGFRB (5q exon 12) fusion gene.

This fusion gene MPRIP-PDGFRB $\mathrm{t}(5 ; 17)(\mathrm{q} 32 ; \mathrm{p} 11)$ was reported in only one other case in $2015 .^{[1]}$ In this article,

*Correspondence: Karolien Beel; Email: karolien.beel@zna.be; Address: Centre of Human Genetics, University Hospitals Leuven and KU Leuven, Leuven, Belgium. 
the authors state that all partner genes of $P D G F R B$ contain coiled-coil domains, which likely cause dimerization and constitutive activation of the tyrosine kinase fusion protein. In the reported patient, imatinib induced a rapid and durable complete remission.

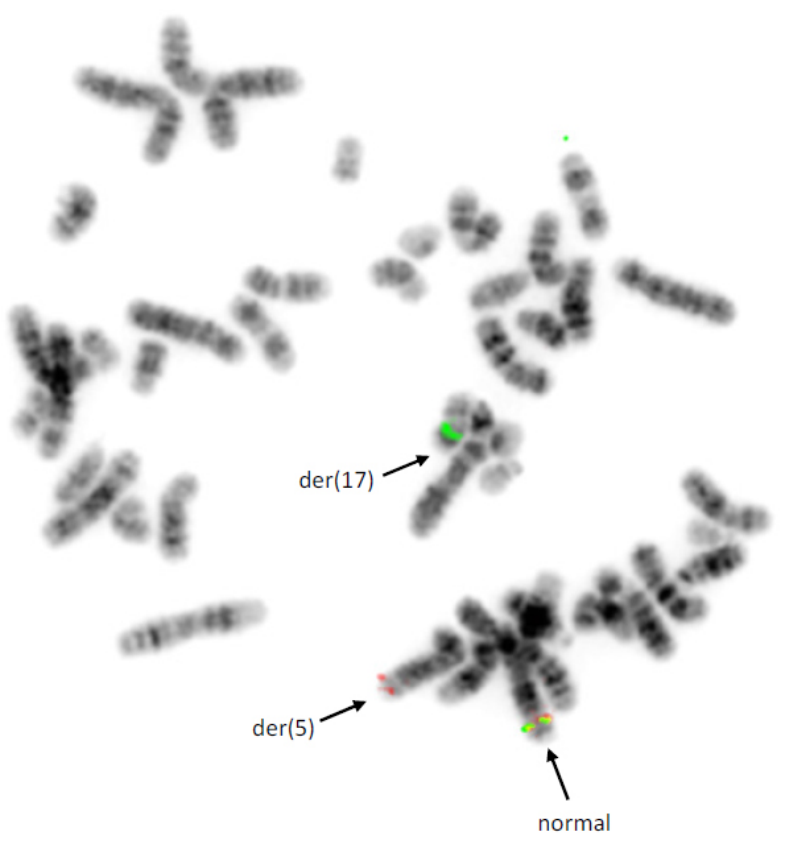

Figure 1. FISH with Vysis LSI PDGFRB break apart probe $^{\mathrm{CE}}$ (Abbott molecular, Illinois, USA)

The normal chr.5 displays a colocalization signal. One 5' telomeric signal was translocated to the der(17), and one 3' centromeric signal remained on the der(5).

Before the introduction of imatinib, the overall survival of patients with a $P D G F R B$ translocation (mostly ETV6-PDGFRB rearrangement) was less than two years, with high mortality due to cardiac damage or leukemic transformation. ${ }^{[2]}$

Our patient was started on imatinib $100 \mathrm{mg}$ daily, which was well tolerated and induced a complete hematological response with normalization of $\mathrm{Hb}, \mathrm{WBC}$, and eosinophil counts after one month.

A personalized molecular test was developed, with diagnostic material of the patient as control. With Primer3 software, a forward primer MPRIP_F TATTGCGGGTAAAGGAATCG, reverse primer PDGFRB_R CCTTCCATCGGATCTCGTAACG, and an internal hybridization probe MPRIP_P FAM - GTACCTGAAACAGGAGATTAGCTC BHQ1 were designed for the MPRIP-PDGFRB fusion gene.

The threshold cycle or crossing point - the cycle at which the amplification plot crosses the threshold - of MPRIP$P D G F R B$ are compared with that of the reference reaction for $A B L 1$ on the same sample (DDCT method) with for- warding primer ABL-F-EAC TGGAGATAACACTCTAAGCATAACTAAAGGT, reverse primer ABL-R-EAC GATGTAGTTGCTTGGGACCCA and internal hybridization probe ABL-P-EAC FAM - CCATTTTTGGTTTGGGCTTCACACCATT - BHQ1.

The result was not corrected for the copy number of $A B L 1$ per cell because the copy number of MPRIP-PDGFRB in the diagnostic sample was unknown. Therefore, a ratio is reported and not a percentage. This ratio is the ratio of the copy number of both genes, assuming that the test amplification of $A B L 1$ and MPRIP-PDGFRB are the same.

No dilutions of the positive control were made to save the diagnostic material of the patient. Sensitivity testing was also not performed, as plasmid DNA, used to clone the MPRIP$P D G F R B$ fragment, could contaminate the sample and because this test is performed in only one patient, due to the uncommonness of this entity.

At diagnosis, an MPRIP-PDGFRB/ABL1 ratio of 25 was reported. After three months of treatment, the $M P R I P$ $P D G F R B / A B L 1$ ratio was 0.076 . From 6 months of treatment with imatinib $100 \mathrm{mg}$ onward, MPRIP-PDGFRB remains undetectable, with a current follow up period of 24 months. The patient is in optimal condition and tolerates imatinib well. Bone marrow FISH after 18 months of treatment revealed no rearrangement of $P D G F R B$, in line with the molecular findings on peripheral blood (see Table 1).

Table 1. Evolution of the MPRIP-PDGFRB/ABL1 ratio

\begin{tabular}{lll}
\hline Time point & Sample & Ratio \% \\
\hline At diagnosis & Bone marrow & 25.0 \\
3 months & Blood & 0.76 \\
6 months & Blood & 0.00 \\
9 months & Blood & 0.00 \\
12 months & Blood & 0.00 \\
18 months & Bone marrow & 0.00 \\
24 months & Blood & 0.00 \\
\hline
\end{tabular}

Tyrosine kinase inhibitor discontinuation is a valid option in a number of CML patients, but it is not yet validated in MPN-eo. A small number of cases with a FIP1L1-PDGFRA translocation have been reported after imatinib cessation, and sustained remissions are noted in less than half of the patients, comparable to CML. Re-initiation of low-dose imatinib remained similarly effective in relapsed patients. Whether the same holds true for PDGFRB constructs remains to be seen.

\section{CONFLiCTS OF InTEREST Disclosure}

The authors declare they have no conflicts of interest. 


\section{REFERENCES}

[1] Naumann N, Schwaab J, Metzgeroth G, et al. Fusion of PDGFRB to MPRIP, CPSF6, and GOLGB1 in three patients with eosinophiliaassociated myeloproliferative neoplasms. Genes Chromosomes Cancer. 2015 Dec; 54(12): 762-70. PMid:26355392. https ://doi .or g/10.1002/gcc. 22287

[2] Marianna D, Nicholas C, Sonja B, et al. Durable responses to imatinib in patients with PDGFRB fusion gene-positive and BCR-ABLnegative chronic myeloproliferative disorders. Blood. 2007 Jan 1; 109(1): 61-4. 